\section{Planning Network Projects in Materials Science and Solar Energy}

\author{
Workshop in Nairobi, Kenya, \\ 21-26 November, 1988
}

Last November, physicists met in Nairobi, Kenya, to discuss development, energy and collaboration and to plan networks in materials science and solar energy. This first workshop in the Third World initiated by the Interdivisional Group on Physics for Development (IGPD) of EPS was organized in response to requests from physicists in the Third World for the establishment of more collaboration projects. The principal aim was therefore not to study the science, but plan concrete projects involving physicists from the North and the South in long term common research programmes.

After a hectic final month of reorganization, moving the workshop from the planned site, Arusha in Tanzania to Nairobi in Kenya, some 50 African physicists met with 14 Europeans and 3 Asians. Invited speakers, from Africa, Asia and Europe, covered fields like Optimization and Field Tests of Photovoltaic Systems, Solar Cell Technology (covering both simple and high technology methods as well as the special problems in Africa) and Measurements of Solar Irradiation. Several talks dealt with different aspects of materials and surface studies with special emphasis on their suitability for solar cells, for absorptive solar cooking as well as for photothermal selective coatings, for example for passive cooling. Several methods for and applications of solar thermal collectors were described with examples of their use.

Ample time had been set aside for parallel sessions dealing with the broad topics of: - Photovoltaic cells

- Solar systems

- Materials characterization

— Field tests of solar systems.

These sessions were used to present ongoing projects in the different institutions, to discuss topics relevant to collaborative research and concretize network project plans.

To date, the workshop has resulted in four major programmes:

- Collaboration between Senegal, Egypt, Thailand, Federal Republic of Germany and France on the study of the performance of special solar equipment in the three different climates represented in the Third World countries; EEC funding proposed. \section{- Collaboration between Tanzania} ("Centre"), Ethiopia, Kenya, Sudan, Uganda, Zambia and Sweden in the field of photovoltaic cells and materials applicable for passive cooling. Extension of collaboration initiated by International Programs in the Physical Sciences, Uppsala.

\section{The Svedberg Laboratory, Uppsala University Post-Doctoral Fellowship in Nuclear Physics}

The Svedberg Laboratory is a national laboratory built around three accelerators, a $6 \mathrm{MV}$ tandem accelerator, a $K=200$ cyclotron for light and heavy ions and a storage and cooler ring (CELSIUS) for proton energies up to $1360 \mathrm{MeV}$ and corresponding energies for heavy ions, e.g. about $400 \mathrm{MeV} /$ nucleon for fully stripped ions with $A \leq 40$. The tandem accelerator has been in operation since 1970. The cyclotron has been in operation for about two years following a major reconstruction; 120 hours of beam per week are delivered on a regular schedule. Proton beams from the cyclotron were recently accelerated to about $600 \mathrm{MeV}$ in the CELSIUS ring. Further tests of the ring are planned for the near future. The cyclotron will be equipped with an external ECR ion source within the next few months. An electron cooling system is being installed in the ring and will be tested during the fall of 1989 .

A fellowship is open to applicants who within the past three years have completed their $\mathrm{PhD}$ lor corresponding) examination in nuclear physics, preferably elementary modes of nuclear excitations such as giant multipole resonances studied at intermediate energies.

A monoenergetic neutron beam facility has been built at one of the beam lines of the cyclotron and an experimental programme to study nuclear structure by means of the $(n, p)$ reaction is in the data-taking stage. Further studies of nuclear reactioons and structure at intermediate energies are being planned.

The fellowship is for one year but can be extended to a maximum of two years. It is free of tax and is worth SEK 120000.- 1 ( US\$ $18000 .-)$ per year to a European or SEK 125000.- 1 ( US\$18700. -1 per year to an overseas physicist. Additional support can be obtained for a fellowship holder with children. Applications with curriculum vitae, publication list and three letters of reference should reach Dr. Leif Nilsson, The Svedberg Laboratory, P.O. Box 533, S-751 21 Uppsala, Sweden

The EUROPEAN SYNCHROTRON RADIATION FACILITY is constructing a state of the art storage ring for $6 \mathrm{GeV}$ electrons and/or positrons to be operated as a high brilliance synchrotron radiation source in the field of X-ray from 1994 on. Financing of the ESRF is shared by 11 European Countries.

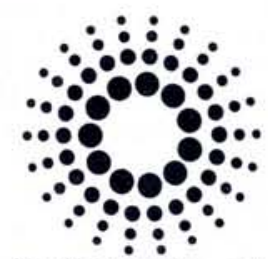

The objectives of the ESRF are to support scientists in the implementation of fundamental and applied research on the structure of condensed matter in fields such as physics, chemistry, crystallography, earth science, biology \& medecine, surface and materials science. The Experiments Division is recruiting in the following fields :

\section{SCIENTISTS}

X-RAY DETECTORS : he/she will be responsible for leading an R\&D programme in X-ray detector systems and for assisting ESRF scientists in the design of detector systems for the beamlines.

Ref. $n^{\circ} 2108$

EXAFS AND ABSORPTION SPECTROSCOPY : He/she will be responsible for design \& construction of one of the beamlines for absorption spectroscopy. Familiarity with instrumentation for EXAFS or XANES in the transmission, fluorescence or electron detection modes is desirable. Close interaction with the Detector Group is expected

Ref. $n^{\circ} 2117$

X-RAY DIFFRACTION : He/she will be responsible for design and construction of one of the crystallography beamlines. Interest and experience in inorganic or protein crystallography are expected.

Ref. $n^{\circ} 2118$

THEORETICAL CONDENSED MATTER PHYSICS : He/she will perform research on theoretical condensed matter physics of relevance for synchrotron radiation research, such as structural properties and electronic structure of solids, surface physics, theory of $\mathrm{X}$-ray processes in condensed matter.

Ref. n²119

Further appointment of scientists in the above or in related fields will follow in the future. Candidates are also invited to apply for these positions.

ESRF scientific appointments are time-limited, up to a maximum of five years. A limited number of permanent appointments is possible in exceptional cases.

The working language in ESRF is English. Knowledge of French is desirable.

ESRF offers you an interesting opportunity in an international atmosphere, and with high technology equipment. Non-French staff benefit from Installation and Expatriation Allowances, and the salaries should be attractive.

If you require more information you can state your name, address under the chosen reference number to us in Grenoble, before Oct. 31 1989, and we shall provide you with an "Application Form" and the information needed.

ESRF - Personnel Office / BP 220 - F 38043 GRENOBLE CEDEX 
- Regional centre for testing and calibration for Solar Energy systems. Possible location: Bujumbura (Burundi), or Bamaku (Mali).

- Project to make an African atlas of solar irradiation by Algeria, France, Ivory Coast and possibly others.

An African Society for Solar Energy, recently established, was given a strong boost during the meeting, as did the realization of an African Journal for Solar Energy. The editor expressed great interest in receiving contributions to the journal from European physicists.

Europhysics News readers should send relevant papers to:

the Editor, Professor E. Kaptouom,

ENS Polytechnique,

B.P. 8390, Yaoundé, Cameroon,

who will also supply more information on the journal.

At the end of the workshop the following declaration was unanimously agreed on:

As scientists working in the field of solar energy who have met in Nairobi, we affirm our intention to continue our research for the benefit of African society. We are pleased that this meeting has already initiated a network of supportive research amongst colleagues from many different countries. Our aim is to develop appropriate forms of energy to satisfy the needs of our societies. We ask that the responsible authorities in Africa give our research the high priority that is needed. Likewise we ask the European authorities to encourage this effort by supporting, as a high priority, cooperative programs of research and development in this field.

Nairobi, 25 November, 1988

IGPD has through this event, organized by scientists in the Third World and an International Programme Committee, found a form of cooperative activity which proved successful, and it is therefore recommended as a guideline for similar endeavours within other fields, or in the same field in different geographical regions. If you are interested, but do not know where to find the contact persons in the Third World, please call on us, and we will do our best to locate candidates. Enquiries should be sent to the

IGPD Secretary, Dr. Jean-Bernard Robert,

CNRS/SNCI, B.P. $166 X$,

38042 Grenoble Cedex, France.

\section{Endre Lillethun Chairman, IGPD}

The first Prize of the High Energy and Particle Physics Division has been awarded to Georges Charpak of CERN one of the pioneers of modern detector systems for his outstanding contributions to experimental particle physics.

The prize, worth 10000 SFR, will be presented during the International Europhysics Conference on High Energy Physics which will take place in Madrid from 6-13 September. On this first occasion it has been donated by the Division, whereas in the future, interest on capital donated by several industrial companies will be the main source.

\section{HEPP Prize}

\section{International Research}

With the rapidity he promised, Ivo Slaus has brought out the Proceedings of the IV EPS Seminar on International Research Facilities held 17-19 March 1989 in Zagreb. Over 520 pages long, the work constitutes a veritable handbook on big physics research facilities now in operation or in an advanced stage of planning throughout the world. Copies have already been mailed to the participants (and to ICTP). Further copies may be ordered from the Secretariat of the European Physical Society in Geneva: price to Individual Members of the Society: 30 SFR; others 60 SFR.

\section{Liquid Physicists Coalesce}

The Liquids Section of the Condensed Matter Division has decided to organize a large scale conference entitled "Liquid Matter". It will take place at the Ecole Normale Supérieure in Lyon, 7-11 July 1990. This will be the first conference of its kind and it is expected that it will be attended by several hundred participants. Its main purpose is to bring together liquid state physicists and chemists by considering all branches of this rapidly growing field. Topics such as statistical mechanics and spectroscopy of simple liquids, physical and physico-chemical hydrodynamics, chaos and turbulence, physics of liquid crystals, liquid polymers, liquid metals, dispersed media, interfacial phenomena and wetting, colloids and microemulsions will be covered. Another community which is addressed is that of scientists and engineers active in industrial research involving liquid state physics and chemistry.

A rapid sketch of the history of this field may be useful to understand the nature of the project. For two centuries,

\section{IGPD}

The aim of the Interdivisional Group on Physics for Development (IGPD) is to encourage more cooperation between physicists with different levels of resources for scientific research both within Europe and the other continents (see also Europhysics News, February 1989, p. 1).

If you find its goals worth your support, you may become a member of the group by indicating this in a note to EPS Main Secretariat, attn. Mme Resegotti, POB 69, CH-1213 Petit-Lancy 2.

Information on your research field will facilitate our calling on you for help in connection with future activities.

Membership in IGPD requires Individual Membership of EPS.

As a member, you will receive the IGPD Newsletter and have the possibility of influencing the development of the IGPD. the term liquid state physics was synonymous with that of fluid mechanics. However, important moves took place at the beginning of this century. Since then the field has opened and changed faster and faster. The first extension was to chemically reacting flows, and the other to liquid crystals, liquid polymers, polyelectrolytes and to dispersed media. A crucial step in the development was the introduction, in the early 50 's, of modern methods of statistical mechanics and of computer simulation. The development of powerful experimental techniques such as neutron or light scattering also gave great impetus to the field. Modern liquid state physics, resulting from all these efforts, is a young and vigorous science. Terms such as disorder, correlations, dissipation, fluctuation and chaos are some of its key words.

Neverthless, in spite of this impressive progress, a structured community of liquid state physicists and chemists is still to emerge. This is the price imposed by the random walk of history and by the separate development of its various sub-disciplines. The scientific activities are not always, and not necessarily, rational. Achievement of the missing unity would be a great step forward. There is no doubt that the EPS offers a particularly suitable frame for such an action, much more so than any individual national physical society. The organizers have thus chosen this route and hope that the creation of a large scale liquid state scientific community will be accelerated by the organization of the "Liquid Matter" Conference in Lyon.

Chairman, Liquids Section of the CMD

\section{S.Bratos}

